\title{
-NOTES-
}

\section{EIGENVALUE BRANCHING CONFIGURATIONS AND THE RAYLEIGH-RITZ PROCEDURE*}

\author{
By J. M. T. THOMPSON (University College, London)
}

Summary. A general theory of elastic post-buckling, applicable to a wide class of structural eigenvalue problems, is developed in generalized coordinates. Attention is restricted to the initial post-buckling path of the structural system on a plot of the load against the critical principal coordinate, and exact first-order solutions for the path are presented. These solutions are compared with the predictions of the non-linear RayleighRitz analysis in which the linear buckling mode is employed as the assumed form, and theorems concerning the results of this analysis are established.

It is seen that the Rayleigh-Ritz analysis will always yield the correct initial slope for the post-buckling path, and that when this slope is zero the analysis will supply an upper bound for the initial curvature. For 'symmetric' systems it is further shown that the Rayleigh-Ritz analysis will always yield the correct initial curvature, while if the curvature is zero the analysis will supply an upper bound for the fourth derivative.

1. Introduction. Experience with thin shell structures has indicated [1] that on encountering any new buckling problem in this field, an attempt must be made to assess the post-buckling characteristics of the structural system. If the load-carrying capacity of the idealized structure is observed to fall as the buckling deformation develops, the lowest critical load may represent a poor and unsafe estimate of the stability limit of a practical imperfect structure.

A quick and reliable procedure for examining the initial post-buckling behaviour of a structure is clearly required, and one such procedure has been developed by Koiter $[2,3]$. This method makes use of the presumably known linear buckling mode, and may involve the solution of a set of linear equations to determine the small initial changes in the buckled form.

A simpler procedure, which however yields less detailed information about the initial post-buckling path, is to use the known linear buckling mode as the assumed form in a non-linear Rayleigh-Ritz analysis. Theorems concerning the results of this procedure are established in the present paper.

A general theory of elastic post-buckling, applicable to a wide class of structural eigenvalue problems is first developed in generalized coordinates, following the lines of an earlier study of elastic instability [4]. First-order solutions are established for the initial post-buckling path of the structural system, and these are subsequently compared with the results of the non-linear Rayleigh-Ritz procedure.

The explicit first-order solutions for the post-buckling path are entirely new, but it should be noted that the theorems concerning the Rayleigh-Ritz procedure are contained implicitly in the more-general theory developed by Koiter [2, 3].

2. General remarks. The sensitivity of the stability limit of an eigenvalue buckling

* Received June 14, 1963; revised manuscript received Nov. 17, 1963. This work was performed at Stanford University with the sponsorship of the National Aeronautics and Space Administration under Grant NsG. 93-60. 
system to initial perturbations is largely determined by the post-buckling characteristics of the idealized perfect system. Thus if no 'small-deflection' post-buckling equilibrium states exist at loads less than the critical load, initial imperfections will usually be insignificant. If on the other hand a post-buckling path emerging from the first branching point yields 'adjacent' equilibrium states at loads less than the critical load, initial inperfections can be expected to yield a marked lowering of the stability limit. The rate at which the load-carrying capacity of the structure decreases along this path will moreover be significant in determining the precise sensitivity of the stability limit.

It is clear from the above remarks that the initial post-buckling behaviour of a structure is of considerable practical importance. If, moreover, the first critical load is discrete, it is apparent that the first-order solution of the corresponding post-buckling path will be of primary interest. Considering the path on a plot of the load against the critical mode amplitude for example, interest will focus in the first instance on the initial slope, while if the slope is seen to be zero, interest will be transferred to the initial curvature.

The theorems of the present paper are restricted to eigenvalue problems exhibiting discrete critical loads, and in the light of the preceding remarks are concerned with the first-order solutions of the initial post-buckling path.

Many structural systems are designed with a high degree of symmetry, and, if the symmetry is preserved by an appropriate choice of coordinates, the post-buckling paths of these systems will consequently be symmetric about the load axis. Two theorems applicable exclusively to a class of symmetric systems are presented, the value of these theorems lying in the fact that the required symmetry of a system can usually be established by a preliminary inspection.

3. Structural system. The general analysis and theorems of the paper are concerned with the post-buckling behaviour of any elastic structure that yields a wellbehaved eigenvalue buckling problem under the influence of a single generalized conservative load. An energy formulation capable of describing any such structural system is developed in the present section.

It is assumed that the deformations of the elastic structure can be analysed into mode-forms, the amplitudes of which will supply a set of generalized coordinates for the structure. It is further assumed that the behaviour of the structure can be described satisfactorily by the use of a large but finite number of coordinates.

Considering then a finite set of $n$ generalized coordinates, $q_{i}$, the strain energy of the structure can be represented by a single-valued function, $U\left(q_{i}\right)$, of these coordinates.

Introducing now a generalized displacement represented by the single-valued function $E\left(q_{i}\right)$, we shall suppose the structure to be loaded by a dead generalized conservative force of magnitude $P$ acting on this displacement, as shown in the schematic diagram of Fig. 1. The total potential energy of the structural system can thus be written as $V=U\left(q_{i}\right)-P E\left(q_{i}\right)$.

We suppose further that the structural system yields a well-behaved pure eigenvalue problem with the trivial undeformed equilibrium state, $q_{i}=E(0)=0$ for all values of $P$. Then we can expand the functions $U\left(q_{i}\right)$ and $E\left(q_{i}\right)$ as power or Taylor series in the generalized coordinates as follows,

$$
\begin{aligned}
& U\left(q_{i}\right)=\frac{1}{2} m_{i j} q_{i} q_{i}+\frac{1}{6} n_{i j k} q_{i} q_{i} q_{k}+\cdots, \\
& E\left(q_{i}\right)=\frac{1}{2} g_{i j} q_{i} q_{i}+\frac{1}{6} h_{i j k} q_{i} q_{i} q_{k}+\cdots .
\end{aligned}
$$




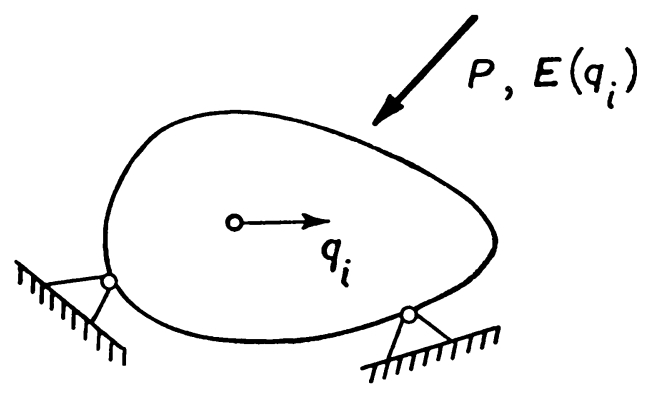

Fig. 1. Structural system.

Here the dummy suffix convention is employed with all summations ranging from 1 to $n$ : the coefficients are constants for a given system, and the sets of coefficients are assumed to be symmetric in the sense that $n_{i j k}=n_{k j i}=n_{j i k}$ etc.

The quadratic form of the strain energy being positive definite, the quadratic forms of $U\left(q_{i}\right)$ and $E\left(q_{i}\right)$ can be simultaneously diagonalized by means of a real non-singular linear transformation of coordinates. If the new (principal) coordinates are $u_{i}$, we then have

$$
\begin{aligned}
& U=\frac{1}{2} u_{i} u_{i}+\frac{1}{6} N_{i j k} u_{i} u_{i} u_{k}+\cdots, \\
& E=\frac{1}{2} G_{i} u_{i}^{2}+\frac{1}{6} H_{i j k} u_{i} u_{j} u_{k}+\cdots,
\end{aligned}
$$

where the positive-definite quadratic form has been normalized. The new coefficients, $G_{i}, N_{i j k}$, etc., are functions of the previous coefficients, $m_{i j}, g_{i j}$, etc.

The total potential energy of the system can now be written as

$$
V=V\left(P, u_{i}\right)=U\left(u_{i}\right)-P E\left(u_{i}\right) .
$$

Thus, focusing attention on the trivial loaded equilibrium state defined by $P=P_{0}$, $q_{i}=u_{i}=0$, and introducing the new symbols,

$$
\begin{aligned}
p & \equiv P-P_{0}, \\
C_{i} & \equiv 1-P_{0} G_{i} \\
D_{i j k} & \equiv N_{i j k}-P_{0} H_{i j k}, \quad \text { etc. }
\end{aligned}
$$

we can finally write the total potential energy function in the form,

$$
V=\left(\frac{1}{2} C_{i} u_{i}^{2}+\frac{1}{6} D_{i j k} u_{i} u_{j} u_{k}+\cdots\right) \quad p\left(\frac{1}{2} G_{i} u_{i}^{2}+\frac{1}{6} H_{i j k} u_{i} u_{j} u_{k}+\cdots\right) .
$$

Equilibrium states of the system are defined by the $n$ equations $\partial V / \partial u_{i}=0$, and the critical loads are given by the $n$ equations,

$$
C_{i}=1-P_{0} G_{i}=0,
$$

that is to say, the critical load associated with the principal coordinate $u_{i}$ is given by $P_{i}=1 / G_{i}$.

We shall assume that the critical loads are discrete, so that $P_{i} \neq P_{i}$ for $i \neq j$, and we shall restrict attention to the first branching point at which the initial stability of the structure is lost.

Assuming then that $P_{1}=1 / G_{1}$ is the first critical load, we shall set $P_{0}=P_{1}$, so 
that $p=P-P_{1}$. The critical principal coordinate is thus $u_{1}$, and at the branching point under consideration the associated stability coefficient is zero, while the remaining stability coefficients are all positive; that is to say, $C_{1}=0$, while $C_{s}>0$ for $s \neq 1$.

The system formulated above and considered in the present paper represents a pure eigenvalue problem, in which the structure suffers no pre-buckling deformation. An example of such a problem is provided by an Euler strut, or by a combination of struts for which the axial flexibilities can be neglected $a$ priori. Since many buckling systems of practical interest do not yield pure eigenvalue problems, an important generalization of the present analysis will be briefly considered.

Starting again with the structural system of Figure 1, a Taylor expansion of $E\left(q_{i}\right)$ about the unloaded state will in general contain linear terms, these terms giving rise to pre-buckling deformations. If the corresponding "fundamental" equilibrium path is given by the single-valued relationships $q_{i}=q_{i 0}(P)$, we can now define changes in the coordinates by the equations $\Delta q_{i} \equiv q_{i}-q_{i 0}$. Defining further a change in the total potential energy

$$
\begin{aligned}
\Delta V & \equiv \Delta U-P \Delta E \\
& \equiv\left[U\left(q_{i}\right)-U\left(q_{i 0}\right)\right]-P\left[E\left(q_{i}\right)-E\left(q_{i 0}\right)\right],
\end{aligned}
$$

this change can finally be expanded as a Taylor series in the $\Delta q_{i}$ and in $p \equiv P-P_{0}$. The linear terms of $\Delta U$ and $\Delta E$ cancelling out, this expansion will contain no linear terms in the $\Delta q_{i}$; and it will in general be non-linear in $p$.

Focusing attention on the first point of bifurcation by equating $P_{0}$ to the critical value of the load, we can now linearize $\Delta V$ with respect to $p$, assuming the point of bifurcation to be well-behaved. Diagonalizing simultaneously the remaining two quadratic forms, the expansion of $\Delta V$ can finally be written in the form of Eq. (3). It follows that the analysis of the present paper is readily generalized to cover systems exhibiting pre-buckling deformations.

4. Initial post-buckling path. We wish now to study the behaviour of the structural system in the vicinity of the first critical equilibrium state. That is to say, we wish to obtain a first-order non-linear solution of the equilibrium equations $\partial V / \partial u_{i}=0$, that is correct in the immediate vicinity of this state.

4.1 Preliminary analysis. In the region under consideration it is clear that $p$ and all the $u_{i}$ will be (vanishingly) small, and that along the initial post-buckling path the critical principal coordinate, $u_{1}$, will be large in comparison with any other principal coordinate, $u_{s}$, where $s \neq 1$.

Thus since $C_{1}$ is equal to zero, and since $G_{1}\left(=1 / P_{1}\right)$ is necessarily non-zero, the equilibrium equation $\partial V / \partial u_{1}=0$ can be written in the form

$$
p / u_{1}=\left(\frac{1}{2} D_{1 i j} u_{i} u_{i}+\frac{1}{6} E_{1 i j k} u_{i} u_{j} u_{k}+\cdots\right)(1+\beta) / G_{1} u_{1}^{2} .
$$

Here and subsequently $\beta$ is used to represent any expression that vanishes in the limit as $p$ tends to zero, and the coefficients $E_{1 i i k}$ are understood to arise from the first series of equation (3).

Further, since $C_{s} \neq 0$ for $s \neq 1$, the equilibrium equation $\partial V / \partial u_{s}=0$ can be written in the form

$u_{s} / u_{1}^{2}=-\left\{\left(\frac{1}{2} D_{s i j} u_{i} u_{i}+\frac{1}{6} E_{s i j k} u_{i} u_{i} u_{k}+\cdots\right)-p\left(\frac{1}{2} H_{s i j} u_{i} u_{i}+\cdots\right)\right\}(1+\beta) / C_{s} u_{1}^{2}$

for all $s \neq 1$. 
4.2 Initial slope. An expression for the initial slope of the post-buckling path is readily derived.

Thus from equation (4) we can immediately write

$$
\lim _{p \rightarrow 0}\left(p / u_{1}\right)=D_{111} / 2 G_{1}
$$

so writing $D_{1} \equiv D_{111}$, we have in all circumstances,

$$
\partial P / \partial u_{1}=D_{1} / 2 G_{1} \text {. }
$$

Here and subsequently it is to be understood that derivatives refer to the post-buckling path at the branching point.

Since, in a mathematical sense, $D_{1}$ is "in general" non-zero, it is seen that a "general" eigenvalue branching configuration will exhibit a finite initial slope on a plot of the load against the critical principal coordinate. Such a branching configuration is associated with an "exchange of stabilities" [3, 4] as indicated by the two configurations of Fig. 2,

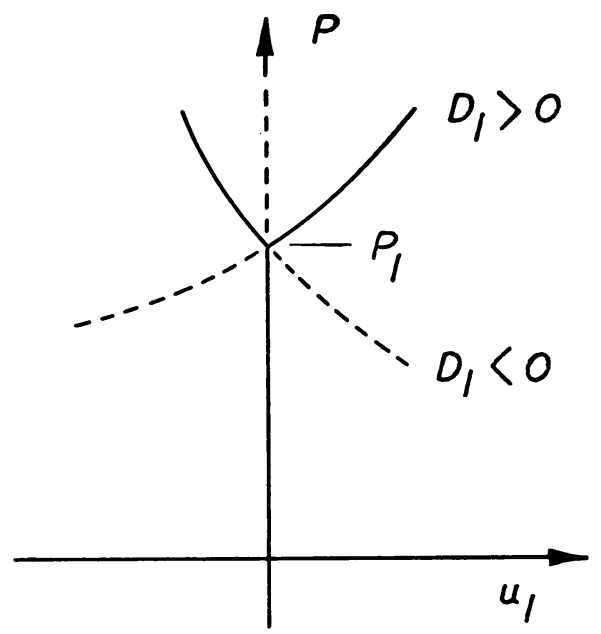

FIG. 2. General eigenvalue branching configurations $\left(D_{1} \neq 0\right)$.

in which a stable path has been represented by a continuous curve, an unstable path by a broken curve.

An Euler strut constrained laterally by a non-linear spring [5], and certain rigidjointed triangular frames [6] exhibit branching configurations of this type.

4.3 Initial curvature. When the initial slope is zero, interest focuses on the initial curvature. We shall thus derive an expression for the curvature under the condition that $D_{1}=0$.

From equation (5) we have

$$
u_{s} / u_{1}^{2}=-D_{s} / 2 C_{s}+\beta
$$

for all $s \neq 1$, where $D_{s} \equiv D_{s 11}$. Then substituting for $u_{s}$ in equation (4) and setting $D_{1}=0$ we have finally

$$
p / u_{1}^{2}=\left(\frac{1}{3} E_{1}-\sum_{2}^{n} D_{s}^{2} / C_{s}\right) / 2 G_{1}+\beta
$$


where $E_{1} \equiv E_{1111}$. Thus in all circumstances for which the initial slope is zero, we have

$$
\partial^{2} P / \partial u_{1}^{2}=\left(\frac{1}{3} E_{1}-\sum_{2}^{n} D_{s}^{2} / C_{\imath}\right) / G_{1} .
$$

Denoting the right-hand side by $T$, we see that $T$ can be either positive or negative, giving the two "non-general" branching configurations of Fig. 3.

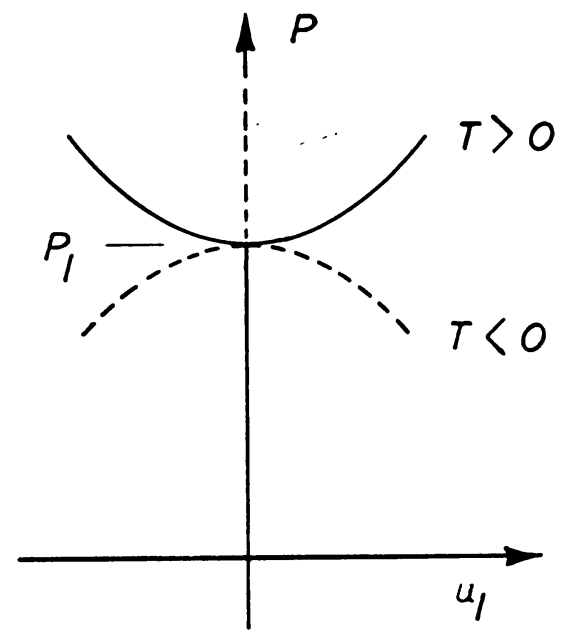

Fig. 3. Non-general eigenvalue branching configurations $\left(D_{1}=0\right)$.

When the curvature is positive, the rising paths are stable [3], an example of this behaviour being the unconstrained Euler strut. When the curvature is negative the falling paths are unstable, and an example of this behaviour is provided by the axially loaded cylindrical panel [7].

4.4 Symmetric system. In many problems of practical interest, the structure and its loads are completely symmetric, so that in a symmetric coordinate system the Taylor expansions for $E$ and $U$ will contain no odd terms. The energy expansion of equation (3) will likewise contain no odd terms, so the coefficients, $D, F, \cdots$, and $H, J, \cdots$, will be identically zero. We shall now examine the initial post-buckling behaviour of such a symmetric system.

Since $D_{1}$ is now identically zero, we see from the previous result, which is of course still applicable, that the initial slope is zero. That is to say, $\partial P / \partial u_{1}=0$, a result which is apparent from the assumed symmetry. Moreover, since all the $D$ coefficients are identically zero, we have from equation (7),

$$
\partial^{2} P / \partial u_{1}^{2}=E_{1} / 3 G_{1} .
$$

The third derivative is zero from the assumed symmetry, and we shall proceed to evaluate the fourth derivative under the condition that the curvature is zero.

When the system is symmetric, Eq. (5) reduces to the form

$$
\because / u_{1}^{3}=-E_{\imath} / 6 C_{\imath}+\beta
$$


for $s \neq 1$, where $E_{s} \equiv E_{s 111}$. Then substituting for $u_{s}$ in Eq. (4) and setting $E_{1}=0$ gives finally,

$$
\partial^{4} P / \partial u_{1}^{4}=\left(\frac{1}{5} G_{11}-2 \sum_{2}^{n} E_{s}^{2} / C_{s}\right) / G_{1},
$$

which is valid for a symmetric system when the initial curvature is zero. Here $G_{11} \equiv$ $G_{11111}$, the latter being understood to arise from the first series of Eq. (3).

5. Rayleigh-Ritz solutions. Let us now examine the solutions that would be obtained for the initial post-buckling path by the use of the Rayleigh-Ritz energy method. It is assumed that the method is used in the context of an appropriate non-linear energy formulation, and that the critical eigenfunction is used as the assumed form, the amplitude of this function being the only free parameter of the analysis.

The energy function, $V\left(p, u_{i}\right)$ of Eq. (3), is contained implicitly within the nonlinear energy formulation, and the assumed form corresponds to the critical principal coordinate $u_{1}$. The energy function of the Rayleigh-Ritz analysis can thus be obtained by setting $u_{s}=0$ for $s \neq 1$ in Eq. (3), and the single equilibrium equation of the analysis can then be obtained by setting $\partial V / \partial u_{1}=0$.

It follows immediately that the Rayleigh-Ritz post-buckling solution is given by

$$
\frac{p}{u_{1}}=\frac{\frac{1}{2} D_{1}+\frac{1}{6} E_{1} u_{1}+\cdots}{G_{1}+\frac{1}{2} H_{1} u_{1}+\cdots},
$$

from which the corresponding first-order solutions are readily derived. These first-order solutions can in fact be obtained from the exact solutions by equating to zero all energy coefficients containing a subscript $s$, where $s \neq 1$.

Since $G_{1}$ is necessarily non-zero, Eq. (10) indicates that $p$ will in all circumstances vanish with $u_{1}$. As is well-known, the proposed Rayleigh-Ritz analysis will thus yield the correct critical load.

The Rayleigh-Ritz solution for the initial slope is seen to be $D_{1} / 2 G_{1}$, which agrees with the exact solution of Eq. (6). The Rayleigh-Ritz procedure will thus yield the correct initial slope for the post-buckling path in all circumstances.

When the initial slope is zero, the Rayleigh-Ritz procedure gives the solution $E_{1} / 3 G_{1}$ for the initial curvature, which corresponds to the first term of the exact solution of Eq. (7). Moreover, since we are considering the first critical load for which $C_{s}>0$ for $s \neq 1$, we see that the second term of Eq. (7), $\sum_{2}^{n} D_{s}^{2} / C_{s}$, can never be negative. Thus since $G_{1}$ is necessarily positive, we see that, when the initial slope is zero, the RayleighRitz solution will yield an upper bound for the initial curvature.

In a similar manner it is readily seen that for a symmetric system the RayleighRitz procedure will always yield the correct initial curvature, and that when the curvature is zero the procedure will yield an upper bound for the fourth derivative.

6. Conclusions. The results of the paper concerning the Rayleigh-Ritz procedure can be summarized in a formal manner as follows.

An elastic structure subjected to a single generalized conservative load is considered. It is assumed that the behaviour of the structure can be described satisfactorily by a large but finite number of generalized coordinates, and that with the use of these coordinates the structural system yields a well-behaved eigenvalue problem lying within the general formulation of Sect. 3. It is assumed that the critical loads of the system are 
discrete, and attention is restricted to the first branching point, at which the initial stability of the structure is lost.

As a special case a symmetric system is considered, for which, with an appropriate choice of coordinates, the strain energy and the deflection of the load are both even functions of the coordinates. The structure of such a system will encounter identical conditions as it buckles in either of the two possible directions, and the post-buckling paths will consequently be symmetric about the load axis.

It is assumed that the linear eigenvalue problem has been solved exactly, so that the linear buckling mode is known, and that an estimate of the initial post-buckling behaviour of the structural system is required. For this estimate, the linear buckling mode can be used as the assumed form in a non-linear Rayleigh-Ritz analysis.

Restricting attention to the branching conditions on a plot of the load against the amplitude of the linear buckling mode, the following results have been established.

Theorem 1. The non-linear Rayleigh-Ritz analysis employing the linear buckling mode will yield the correct initial slope for the post-buckling path under all circumstances.

Theorem 2. When the initial slope is zero, the non-linear Rayleigh-Ritz analysis employing the linear buckling mode will yield an upper bound for the initial curvature of the post-buckling path.

Theorem 3. The non-linear Rayleigh-Ritz analysis employing the linear buckling mode will yield the correct initial curvature for the post-buckling path of a symmetric system.

Theorem 4. When the initial curvature is zero, the non-linear Rayleigh-Ritz analysis employing the linear buckling mode will yield an upper bound for the initial fourth derivative of the post-buckling path of a symmetric system.

\section{REFERENCES}

1. Y. C. Fung and E. E. Sechler, Instability of thin elastic shells, Proc. First Symposium on Naval Structural Mechanics, Pergamon Press, 1960

2. W. T. Koiter, On the stability of elastic equilibrium, Dissertation, Delft, 1945

3. W. T. Koiter, Elastic stability and post-buckling behaviour, Nonlinear Problems, University of Wisconsin Press, 1963

4. J. M. T. Thompson, Basic principles in the general theory of elastic stability, J. Mech. Phys. Solids $11,(1963) 13$

5. H. S. Tsien, Buckling of a column with non-linear lateral supports, J. Aeron. Sci., 9, (1942) 119

6. S. Britvec, Ph.D. dissertation, Department of Engineering, Cambridge University, 1960

7. W. T. Koiter, Buckling and post-buckling behaviour of a cylindrical panel under axial compression, Trans. Natl. Aeron. Research Institute, Amsterdam, Report S476, 1955 